\title{
An Analysis of Image Enhancement Techniques for Dental $\mathrm{X}$-ray Image Interpretation
}

\author{
Siti Arpah Ahmad, Mohd Nasir Taib, Noor Elaiza Abdul Khalid, and Haslina Taib
}

\begin{abstract}
This paper presents qualitative and quantitative comparison between original images and four image enhancement techniques namely adaptive histogram equalization (AHE), contrast adaptive histogram equalization (CLAHE), median adaptive histogram equalization (MAHE) and sharp contrast adaptive histogram equalization (SCLAHE) applied to dental $x$-ray images. Dental $x$-ray images usually taken with low radiation dosage are often presented as dark, low in contrast and noisy. These problems are usually solved with image enhancement techniques. However, choosing an appropriate technique is not an easy task especially for the purpose of disease diagnosis of periapical related lesion. This research involves the collection of ten intra-oral dental $x$-ray images which were collected from the Faculty of Dentistry UiTM Shah Alam, Malaysia. Each of the enhancement methods is applied to every collected image. A dentist was then asked to do the evaluation using questionnaire. She graded the quality of the images and the diagnostic ability of the periapical pathology observed in the images. Subsequently the quantitative analysis using contrast improvement index (CII), signal to noise ratio (SNR) and root mean square error (RMSE) were done to investigate the characteristic of the images base on the dentist evaluation. The finding shows that the enhancement techniques managed to enhance the pathology slightly better than the original image.
\end{abstract}

Index Terms-Contrast enhancement, adaptive histogram equalization (AHE), dental X-ray.

\section{INTRODUCTION}

Presently, the first line of disease diagnosis via imaging modalities relies mostly on x-ray images even though other modalities such as Magnetic resonance (MRI) and computer tomography (CT) provide more accurate interpretations [1]. This is also true for the field of dentistry where $\mathrm{x}$-ray images are consistently used to assist dentists in deciding the appropriate treatment for patients [2]. Commonly encountered periapical diseases are granulomas and cysts which occur in the jaw area. Other periapical diseases are

Manuscript received May 1, 2012; revised May 31, 2012. This work was supported in part by the Ministry of Science, Technology and Innovation (MOSTI), Malaysia, under e-science fund (06-01-01-SF0306) and Dana Kecemerlangan fund, Research Management Institute (RMI), Universiti Teknologi MARA Malaysia (UiTM), (600-RMI/ST/DANA 5/3DST(42/2009).

Siti Arpah Ahmad and Mohd Nasir Taib are with Faculty of Electrical Engineering, Universiti Teknologi MARA, Shah Alam, 40450, Malaysia (e-mail: arpah@tmsk.uitm.edu.my; dr.nasir@ ieee.org).

Nor Elaiza Abdul Khalid with Faculty of Computer and Mathematical Science, Universiti Teknologi MARA, Shah Alam, 40450, Malaysia (e-mail: elaiza@tmsk.uitm.edu.my).

HaslinaTaib is with School of Dental Sciences, Universiti Sains Malaysia, Health Campus, Kubang Kerian Kelantan, Malaysia (e-mail: haslina@kck.usm.my). lesions such as non-odontogenic and malignancies [3]-[4]. The pathologies such as periapical radiolucency, widen periodontal ligament space and loss of lamina dura are the common abnormalities that related to the disease [1][1]. Sometime these diseases can obviously be detected in an $\mathrm{x}$-ray but on other occasion they can be missed since x-ray images are often very noisy and low in contrast[5]-[6]. The nature of intra-oral digital radiographs is low image quality due to low dose usage. The procedure of low dose usage is related to its effect to patient's health [7]-[8]. Thus image processing techniques such as image enhancements methods are the accepted methods to enhance the radiograph's image quality[5]-[6],[9]. One of the parameter to apply image enhancement is by manipulating the image contrast [10]. Contrast enhancement techniques such as histogram equalization (HE), adaptive histogram equalization (AHE), and contrast limit adaptive histogram equalization (CLAHE) have been widely used to improve the contrast in medical images [5][11]-[13]. For example, HE and AHE methods had been used to enhance fish bone impaction in the soft tissue of lateral neck radiograph[2]. AHE and CLAHE algorithms were applied on mammograms for breast cancer [12]-[15] as well as dental radiographs [2],[16]-[22]. Application of image enhancement techniques to dental $\mathrm{x}$-ray images shows an active ongoing research [16]-[22]. Enhancement techniques that had been reported in the literatures were high-pass filters and contrast enhancement. High pass filter types that had been used are shadow [18] and sharpening [18][22]. The pathologies that had been investigated were periodontal ligament space [21], lamina dura [2] and condition of alveolar bone [19].

The main type of enhancement technique used in this work is AHE. AHE is originated from histogram equalization (HE) technique. $\mathrm{HE}$ is an algorithm that improved the visual appearance of the image by assigning each pixel a new intensity value based on previous intensity level by using Equation I [5].

$$
G_{i}=\left[\sum_{j=0}^{i} N_{j}\right]\left(\frac{\max \text { IntensityLevel }}{\text { NoofPixels }}\right)
$$

However, because HE operates in global contrast of the image, the disadvantage is that it may increase the contrast of background noise and resulting in lower usable signal [23]. Due to that AHE is introduced by multiple local window size area, emphasizes local contrast rather the overall contrast [5].

Though, AHE drawbacks was that its operation resultant wash out effect [24], introduces artifacts [25] and losing out the image details [26]. Consequently, the Contrast Limit Histogram Equalization (CLAHE) is created by limiting the local contrast-gain by restricting the height of local histogram 
[12]. Still CLAHE problem is linked to high contrast in both foreground and background increasing the visibility of the main mass at the cost of simultaneously creating small yet misleading intensity in homogeneities in the background [13].

All these drawbacks encourage the combination of AHE and CLAHE with other algorithms [11],[24],[27]-[31]. Sharpening and median filter is often combined with classic contrast enhancement methods [29][31] to enhance medical images [24],[29],[31].

Quantitative measurements such as CII, SNR and RMSE are accepted in the field of image processing to evaluate the performance of proposed techniques [5],[11],[16]-[17],[32]-[35]. All of these measurements were usually used in medical field by the radiologists to assist their interpretation of x-ray images [32]. Most of the proposed techniques were application of wavelet analysis [32]-[35] towards mammographic and ultrasound [11] images.

The aim of this paper was to compare the diagnostic ability between original and enhanced images. While for the enhanced images, the focus of comparison is the quantitative measurement between the standard image enhancement techniques (AHE and CLAHE) with the purposed algorithms (SCLAHE and MAHE).

\section{MATERIAL AND METHODS}

\section{A. Input Images}

Ten intra-oral digital radiographs were taken from Faculty of Dentistry, UiTM Shah Alam, Malaysia. The X-ray machine used was Planmeca Intra-oral x-ray machine.

Fig.1 shows one of the original samples of the radiographic images and its corresponding global histogram. As clearly observed, the gray level distribution is highly skewed towards the darker site. The histogram distribution could be stretched to cover the whole gray scale instead dominantly towards the skewed site. For each of the original images, four enhanced images namely, AHE, CLAHE, MAHE and SCLAHE were produced to make a total of sixty images for visual assessment by a dentist.

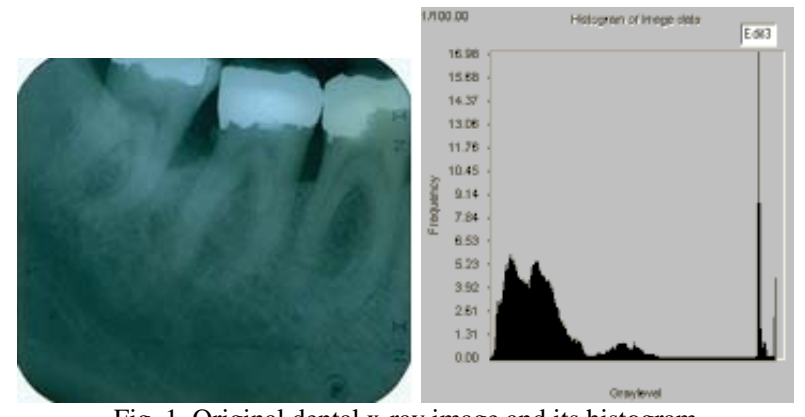

Fig. 1. Original dental x-ray image and its histogram

\section{B. Application of Image Enhancement Techniques}

AHE is used to improve contrast based on local histogram unlike the global contrast enhancement which uses the histogram for the entire image. AHE divides the image into several non-overlapped sub-images and derives their histograms. Then, it modifies the histogram to enhance the contrast of the pixels within the sub-images [5]. Fig.2 shows the stages of the AHE processing for this work. The stages start from the original image with its histogram. Next, the images are masked into 3X3 matrix and then each of the sub-windows will be applied with the AHE technique. After that the whole image is interpolated [10].

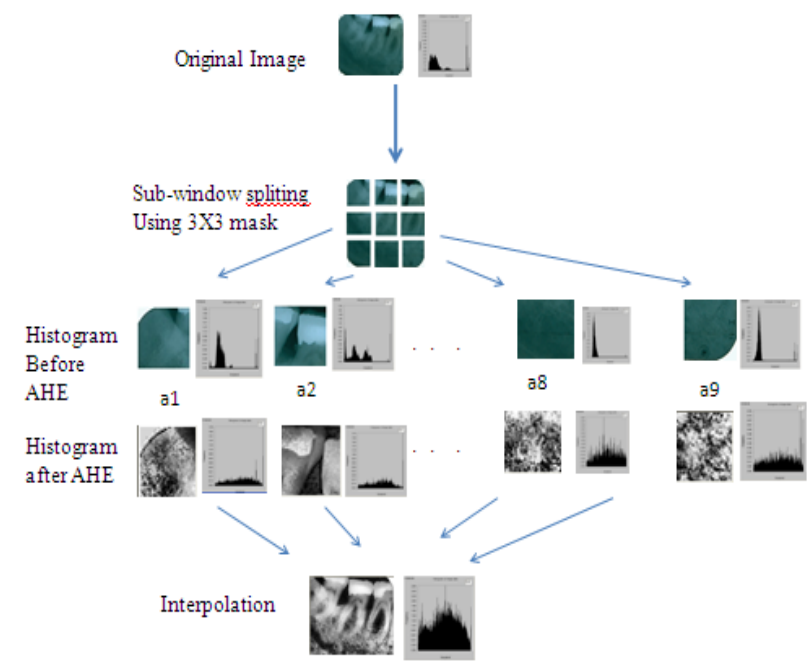

Fig. 2. Stages of AHE processing

MAHE technique combines, median filtering and AHE methods. Median filter is introduced as one of its pre-processing technique to reduce noise and identify its impact towards the contrast of the dental images. This technique updates the pixel value in ascending order. The centre pixel in the $3 \times 3$ area is then replaced with the median value [11].

SCLAHE consists of combination of image sharpening and contrast limited adaptive histogram equalization (CLAHE). The purpose of using the sharpening filters is to sharpen the outline of the periapical features. This work utilized Laplacian filter to perform image sharpening process. Laplacian detects the outlines of the objects by convolving a mask with a matrix centered on a target pixel. The Laplacian detects the edge using a mask as in Fig.3[12].

\begin{tabular}{|l|l|l|}
\hline & -1 & \\
\hline-1 & 4 & -1 \\
\hline & -1 & \\
\hline
\end{tabular}

Fig. 3. Laplacian Edge Detection

CLAHE reduces the problem associated with AHE in relation to noise. This technique limits the slope associated with the pixel range to prevent saturation [23]. It can be an AHE or HE by choosing the appropriate contrast factor. CLAHE is to prevent the random noise introduced during local Histogram Equalization by limiting the maximum slope of the greyscale transform function. The slope of the cumulative distribution function is determined by the bin counts. If a bin has a large count, it will result in more slopes. By thresholding (clipping) the maximum histogram count, we can ensure a limit on the slope [12]. The approach used in the CLAHE algorithm is to clip histograms exceeding the threshold and redistribute the excess evenly among all the remaining bins. Slope value used in the program should be greater to ensure more contrast. Smaller slope will limit the contrast more and hence serve to reduce noise [23]. 


\section{The Visual Assessment}

The visual assessment was done using questionnaire. The images were visually inspected by a dentist on the computer monitor. Each of the images was arranged in random to avoid bias. The questions as well as the images also provided in hardcopy for the dentist to write in the assessment.

There are four stages of evaluations. First, the dentist had to evaluate the present of periapical radiolucency (PR) abnormality. Then assess the present of widen periodontal ligament space (widen PDLs) abnormality. Third was decided whether loss of lamina dura (loss of LD) abnormality exists. Then the qualities of the images were judged based on her experience. The grades for abnormalities assessment were scale 1 ; abnormality detected 2; no abnormality detected but other abnormality detected and 3; no specify abnormality detected and no other abnormality detected. The grades for image quality were scale from 1 (condition that the quality of image is poor and periradicular not read) to 5 (condition that the quality of image is very good and periradicular can be read).

\section{Quantitative Evaluation}

The quantitative evaluations were done using contrast improvement index (CII), signal to noise ratio (SNR) and root mean square error (RMSE). All of these measurements depend on the pixel values of the images.

The CII is produced by the following equation [32]-[36];

$$
C I I=\frac{\text { Cprocess }}{\text { Coriginal }}
$$

where both are the contrast value for the region of interest in the processed and original images respectively .The $\mathrm{C}$ value is derived from the following;

$$
C=\frac{(f-b)}{(f+b)}
$$

where $f$ is the mean gray-level value of the image and $b$ is the mean gray-level value of the background [36].

SNR is the statistical value to measure signal out of noise in an image by the ratio of the signal standard deviation to the noise standard deviation [37]. The formula of SNR is as follows;

$$
S N R=10 \log _{10}\left[\frac{\sum_{0}^{n_{x}-1} \sum_{0}^{n_{y}-1}[r(x, y)]^{2}}{\sum_{0}^{n_{x}-1} \sum_{0}^{n_{y}-1}[r(x, y)-t(x, y)]^{2}}\right]
$$

RMSE it is used to measure the average magnitude of error in the enhanced image based on the original image. It quantifies the average sum of alteration in each pixel of the enhanced image and portrays the average change in a pixel caused by the SAHE and SCLAHE [38]. Lower the RMSE value indicates better enhancement [11].

$$
R M S E=\sqrt{\frac{1}{n_{x} n_{y}} \cdot \sum_{0}^{n_{x}-1} \sum_{0}^{n_{y}-1}[r(x, y)-t(x, y)]^{2}}
$$

\section{RESULTS}

Results are presented in the following manners; Firstly, the distribution between grades (grade $=1$, grade $=2$ and grade $=3$ ) of original images and the CEAs were reported. Second, the results for grade $=1$ only for pathologies abnormality will be presented. Third is about the image quality. Finally, the quantitative results will be produced

Fig.4. shows the distribution between grades. At glance, it displays that grade $=1$ were the majority for original and the image enhancement techniques. Grade 2 and 3 were minority.

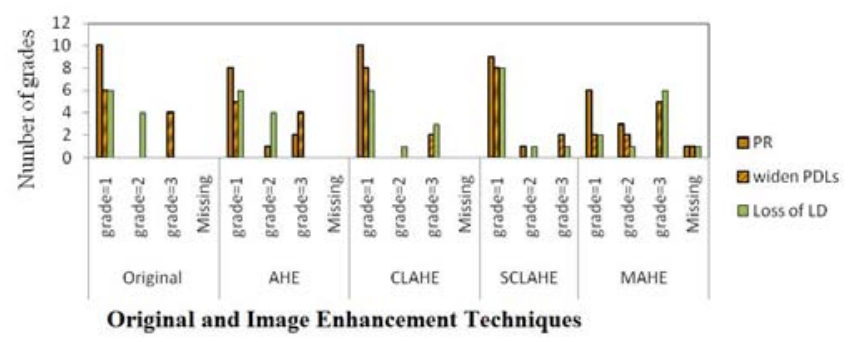

Fig. 4. Distribution dentist's grade between original and image enhancement techniques

Fig.5. shows the results of clearly detected abnormalities only. The figure shows that for PR abnormality, Original and CLAHE were able to detect the abnormality the most (10). Secondly goes to SCLAHE (9). The last were AHE, followed by MAHE. For widen PDLs abnormality, the figure display that, CLAHE and SCLAHE (8) able to overcome original images (6). The ranking then goes to AHE (5) and MAHE (2). Loss of LD indicates that SCLAHE (8) able to overcome others.

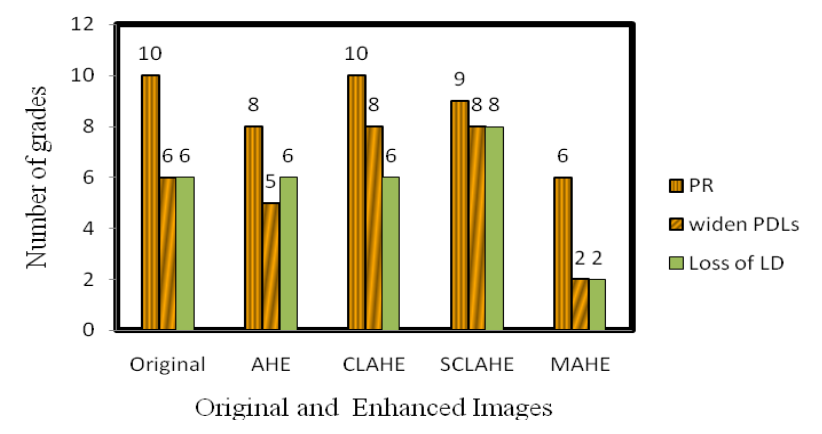

Fig. 5. Grade $=1$ for all abnormality detection.

Fig.6. shows the image quality assessment. Out of five scales, the dentist only grade $=1$ (condition that the quality of image is poor and periradicular not read) and grade $=2$ (condition that the quality of images is acceptable and periradicular can be read). However, only CLAHE method able to get grade $=3$ (condition that the quality of images is good and periradicular can be read). Focusing on grade=2, it shows that SCLAHE (10) able to overcome original (8) images.

Fig.7. displays the CII, RMSE and SNR of the images. It shows that the pattern for AHE and MAHE were the same. The trend was, CII and SNR values were lower but the RMSE is higher. CLAHE and SCLAHE display the same trend where all of the values were almost the same. 


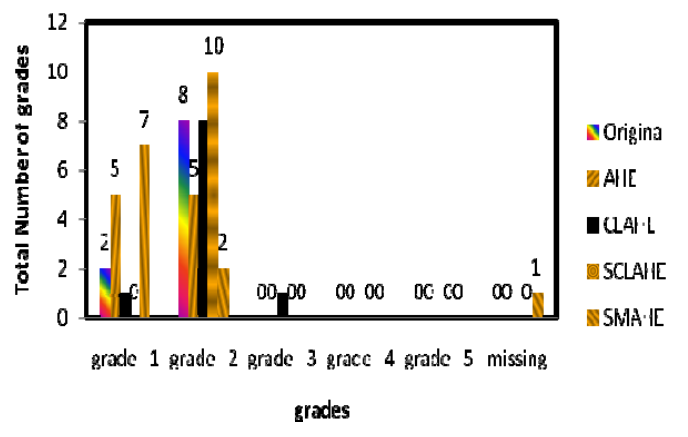

Fig. 6. Image quality of the image samples.

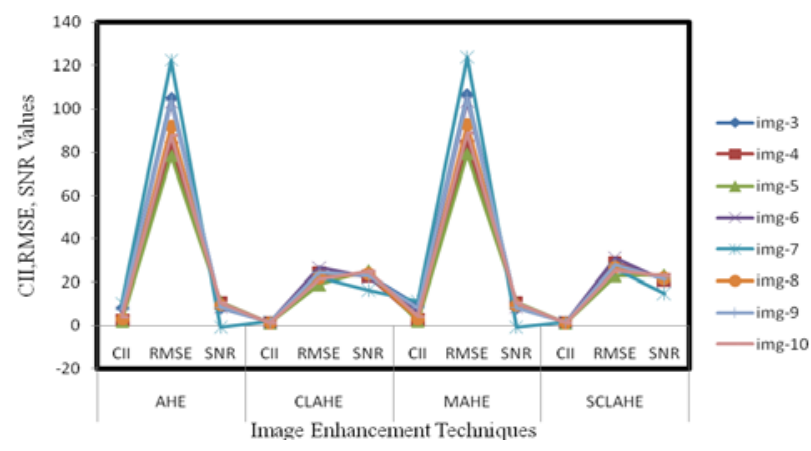

Fig. 7. Overall values of CII, RMSE and SNR for all the images.

Fig.8. was constructed from dentist assessment for the images that have grade $=1$ only. Then the minimum, maximum and average values of CII, SNR and RMSE were analyzed. The trend shows that, CII, SNR and RMSE values for all the three abnormality were consistent. The average values for CII seem to be the lowest (2.72-2.77). Average SNR values were ranging from 6.65 to 18.31 . Finally averages of RMSE values were between 2.77 to 48.88 .

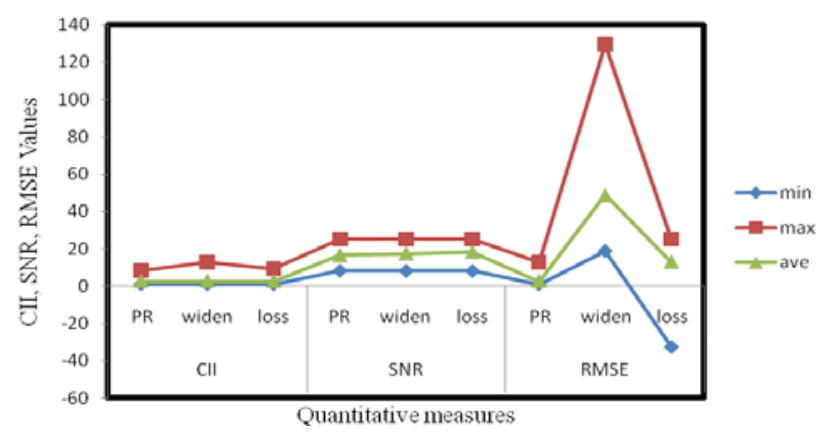

Fig. 8. Comparison between minima, maxima and average values of CII, SNR and RMSE of the abnormalities.

Since average values were not seems to represent the overall pattern especially for RMSE values Fig.9 was produced. The figure was formed from the median values of CII, SNR and RMSE of the abnormalities. It shows that, for PR abnormally, the CII, SNR and RMSE values are ranging from 16.6 to 18.8. For widen PDLs, the values were ranging from 1.67, 18.42 and 27.33. Finally for Loss of LD abnormality, values are ranging from 1.65, 21.18 and 27.92. CII values for widen PDLs and Loss of LD abnormalities, have similar values. But for PR abnormality, the CII value was the highest. For SNR values, it shows that PR abnormality get the lowest values, follow by widen PDLs and the uppermost is Loss of LD abnormality. RMSE shows the maximum values compares to others with widen PDLs and Loss of LD abnormalities were almost the same. However, for PR abnormality, the value is almost similar to CII value.

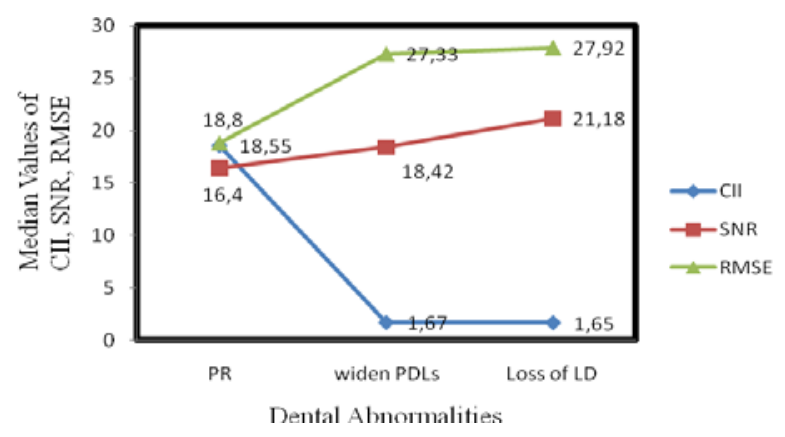

Fig. 9. The Quantitative values of dental abnormalities

\section{Discussion}

This work examined the performance of image enhancement techniques compare to the original images. The assessments are based on subjective evaluation as well as statistical measurement using CII, SNR and RMSE.

In ability to detect the abnormalities, it seems that CLAHE is at par with original images in detecting PR abnormality. However, for widen PDLs and Loss of LD abnormalities, image enhancement techniques able to overcome original images (Fig.5.). Looking at the image quality results, it shows that, dentist score only goes to grade $=1$ and grade $=2$ only (Fig.6.), but CLAHE able to get grade $=3$. This finding is in line with previous work [2], [18]-[20] claimed that image enhancement techniques do increase the image quality and diagnostic ability.

In the specific domain such as in dentistry, the expert (the dentist) is the ultimate judge [10] in doing the interpretation. Due to that, our work gives priority to the dentist as the expert by taking her grade's as reference. Based on the results (Fig.5), we calculated CII, SNR and RMSE values of all the images. The results show a pattern that, AHE and MAHE had similar pattern with RMSE get highest values. CLAHE and SCLAHE shows lower value of RMSE, but CLAHE get the lowest (Fig7.). Focusing on CII values, it is claimed the highest values is the better [32], but our results show that MAHE and AHE get the highest CII, but also get high RMSE is high. The best combination goes to CLAHE and SCLAHE where they get lower RMSE (Fig.8).

\section{CONCLUSION}

In conclusion, our work support that image enhancement do contribute to the better appearance of the images, therefore improve the diagnostic ability in intra-oral digital images.

\section{REFERENCES}

[1] T. Ozen, A. Cebeci, C. S.Paksoy "Interpretation of chemically created periapical lesion using 2 different dental cone-beam computerized tomography units, and intraoral digital sensor, and conventional film”, Oral Surg. Oral Med. Oral Pathol. Oral Radiol. Endod, Vol 107, No.3, pp: 426-432, 2009. 
[2] M. Mehdizadeh and S.Dolatyar, "Study of Effect of Adaptive Histogram Equalization on Image Quality in Digital Preapical Image in Pre Apex Area”, research Journal of Biological Science”,pp: 922 - 924, vol: 4, issue: 8, 2009.

[3] J. A.Regezi, "Periapical Disease: Spectrum and Differentiating Features”, Journal of The California Dental Association”, 1999

[4] O.E.Langland, R.P.Langlais and J.W.Preece, ”Principles of Dental Imaging”, Lippincott Williams \& Wilkins, 2002.

[5] N. M.Noor, N. E. A.Khalid M. H. Ali and A. D. A. Numpang,"Enhancement of Soft Tissue Lateral Neck Radiograph with Fish Bone Impaction Using Adaptive Histogram Equalization(AHE),The $2^{\text {nd }}$ International Conference on Computer Research and Development,2010.

[6] N. E. A.Khalid, N. E., Manaf, M., Aziz, M. E., \& Ali, M. H. (2007, Nov. 25-28). CR images of metacarpel cortical edge detection-bone profile histogram approximation method. Intelligent and Advanced Systems, 2007. ICIAS 2007. , 702-708.

[7] M. Sakata and K. Ogawapp, "Noise Reduction and contrast enhancement for small-dose X-ray images in wavelet domain," presented at the Nuclear Science Symposium Conference record (NSS/MIC), 2009.

[8] R. Aufrichtig and P. Xue, "Dose efficiency and low-contrast detectability of an amorphous silicon x-ray detector for digital radiography," Phys. Med. Biol., vol. 45, pp. 2653-2669, 2000.

[9] R. B. Tiwari, "Dental X-ray Image Enhancement based on Human visual system and local Image statistic" Proceeding of The International Conference of Image Processing, Computer Vision and Pattern Recognition, pg: 100-106, Vol. I\&II, 2006.

[10] R.C.Gonzales and R.E.Woods, Digital Image Processing Second Edition: Prentice Hall, 2002.

[11] K. Thangavel, R. Manavalan, I. L. Aroquiaraj, "Removal of Speckle Noise from Ultrasound Medical Image based on Special Filters: Comparative Study”, ICGST-GVIP Journal, Vol.9, Issues(III), pp: 25-32. 2009

[12] E. D. Pisano, "Contrast Limites Adaptive Histogram Equalization Image Processing to Improve the Detection of Simulated Spiculations in Dense Mammograms," Journal of Digital Imaging, vol. 11, pp. 193-200, 1998

[13] P. Rahmati, G.Hamarneh, D. Nussbaum and A. Adler, " A New Preprocessing Filter for Digital Mammograms, Lecture Notes in Computer Science,vol: 6134, pp: 585-592, 2010.

[14] Y. Jusman, N. A. M.Isa and R. Kurnia, "A Proposed System for Edge Mammogram Image", Proceeding of the $9^{\text {th }}$ WSEAS International Conference on Application of Electrical Engineering”, pp: 117 - 123, 2010.

[15] A. E. Hassanien and J.M.Ali, "A Fuzzy-Rule Based Algorithm for Contrast Enhancement of Mammograms Breast Masses," presented at the 4th WSEAS International Conference Information Science, communication and application, , 2004.

[16] S. A. Ahmad, M. N. Taib, N. E. Khalid, R. Ahmad and H. Taib, "The Effect of Sharp Contrast-Limited Adaptive Histogram Equalization (SCLAHE) on Intra-oral Dental Radiograph Images, ," presented at the 2010 IEEE EMBS Conference on Biomedical \& Sciences (IECBES 2010). 2010.

[17] S. A. Ahmad, M. N.Taib, N. E. Khalid, R. Ahmad and H. Taib"Analysis of Compound Enhancement Algorithms (CEA) based on Adaptive Histogram Equalization (AHE) on Intra-oral Dental Radiographs Images," International Journal of New Computer Architectures and their Applications (IJNCCA), vol. 1, No4, pp: , 2011, 2011.

[18] BG.Baksi, E. Alpz, E. Sogur and A. Mert,” Perception of anatomical structures in digitally filtered and conventional panoramic radiographs: a clinical evaluation”, Dentomaxillofacial Radiology, vol.39, pp: 424-430, 2010.

[19] W. G. W. Alves, E. Ono, J. L. O. Tanaka, E. M.Filho, L. C. Moraes, M. E. L. Moraes and J. C. M. Castilho, "Influence of image filters on the reproducibility of measurements of aveolar bone loss", Journal of Applied Oral Science, Vol. 4(6), pp: 415 - 420, 2006.

[20] T. Sund and A. Moystad,"sliding window adaptive histogram eqaulization in intraoral radiographs: effect on image quality", Dentomaxillofacial Radiology, Vol.35, pp.133-138, 2006.

[21] S. Yalcinkaya, A. Kunzel, R. Willers and J. Becker, "Subjective image quality of digitally filtered radiograph acquired by the Durr Vistascan system compared with conventional radiographs," Oral Surg Oral Med. Pathol. Oral Radiol. Endod, vol. 101, pp. 643-651, 2006.

[22] F. Gijbels, A. M. D. Meyer, C. B. Serhal, C. V. D. Bossche, J. Declerck, M. Persoons, R. Jacob "The subjective image quality of exact digital and conventional panoramic radiography" Journal of Clinical Oral
Investigation, Vol.4, pg.162 - 167, 2000.

[23] M. A. J.Poulist. (2012, 17th May 20102). Contrast Limited Adaptive Histogram Equalization(CLAHE). [online]. Available: http://radonc.ucsf.edu/research_group/jpouliot/Tutorial/HU/Lesson7.h $\mathrm{tm}$

[24] W. Zhiming and T. Jianhua, "A fast implementation of Adaptive Histogram Equalization," presented at the 8th International Conference on Signal Processing (ICSP), 2006.

[25] T. C.Jen and S.Wang, "Histogram Equalization Based on Local Characteristics," presented at the Proceedings of the 2005 Workshop on Consumer Electronics and Signal Processing (WCEsp2005), 2005.

[26] T. Jen, S. Wang, "Generalized Histogram Equalization Based on Local Characteristics," presented at the 2006, IEEE International Conference on Image processing, 2006.

[27] H. Yoon,Y. Han and H. Hahn, "Image Contrast Enhancement based on Sub-histogram Equalization Techniques without Over-equalization Noise," World Academy of Science, Engineerng and Technology, pp. 176-182, 2009.

[28] P. Jagatheeswari, S. Suresh Kumar, M. Rajaram, "Contrast Enhancement Based on Histogram Equalization Followed By Median Filter," presented at the Proceeding of the International Conference on Man-machine System (ICoMMS), Batu Ferenggi,Penang Malaysia, 2009.

[29] T.A.Mahmoud and S.Marshall, "Medical Image Enhancement using Threshold Decomposition Driven Adaptive Morphological Filter," presented at the 16th European Signal processing Conference (EUSIPCO) Laussane, Swizerland, 2008.

[30] R. Kimmel. R. Malladi and N. Sochen, "Images as Embedded Maps and Minimal Surfaces: Movie. Color, Texture and Volumetrics Medical Images", International Journal of Computer Vision, Vol: 3992), 111-129, 2000

[31] T. Kitasaka, K. Mori, J. Hasegawa and J. Toriwaki,”A Method for Extraction of Bronchus Regions from 3D Chest X-ray CT Images by Analyzing Structural Features of the Bronchus”,FORMA, Vol:17,No. 4, pp: 321- 338, 2002.

[32] J. H. Yoon and Y. M. Ro, "Enhancement of the Contrast in Mammographic Images Using the Homomorphic Filter Method," IEICE TRANS. INF \&SYST, vol. E85-D, 2002.

[33] M. R. Hoque and M. R. Mahfuz, "A New Approach in Spatial Filtering to Reduce Speckle Noise," International Journal of Soft Computing and Engineering (IJSCE), vol. 1, pp. 29-32, 2011.

[34] W. Dabour, "Improved Wavelet Based Thresholding for Contrast Enhancement of Digital Mammograms," presented at the 2008 International Conference on Computer Science and Software Engineering, 2008.

[35] H. Kyung, N. N. Thanh, S. M. Kim and Y. M. Ro, "Robust Contrast Enhancement for Microcalcification in Mammography," in Computational Science and Its Applications (ICCSA) 2004, 2004.

[36] I. N. Bankman, "Handbook of Medical Image Processing and Analysis," ed, 2009.

[37] J. Sijbers, P. Scheunders, N. Bonnet, D. V. Duck and E. Raman, "Quantification and Improvement of the Signal-to-Noise Ratio in a Magnetic Resonance Image Acquisition Procedure," Magnetic Resonance Imaging, vol. 4, pp. 1157-1163, 1996.

[38] A. M. Aibinu, M. J. E. Salami, A. Shafie, M. Ali and I. A. Bamgbopa, "Assessment of Mould Growth on Building Materials using Spatial and Frequency Domain Analysis Techniques," International Journal of Computer Science and Network Security, vol. 9, pp. 154-167, 2009.

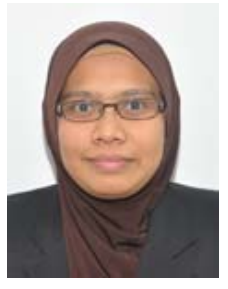

Siti Arpah Ahmad has received BSc (Hon's) in Information Technology from Universiti Utara Malaysia in 1997 and M.Sc in Electrical Engineering from University of The Ryukyus, Japan in 2002. Currently is pursing $\mathrm{PhD}$ in Faculty of Electrical Engineering, Universiti Teknologi MARA, Shah Alam. Her current research interests are in Image Processing, Biomedical, Computer Networking and Parallel Computing

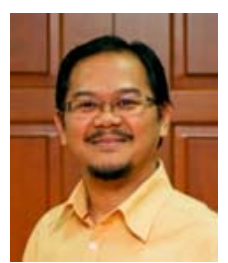

Mohd Nasir Taib has received B.Eng.(Electrical) from Univ. of Tasmania, Australia, MSc (Control System) with Distinction from Univ. Of Sheffield, UK and $\mathrm{PhD}$ (Control \& Instrumentation), UMIST, UK. Currently he is a Professor and Dean of the Faculty of Electrical Engineering, Universiti Teknologi MARA. His Professional Memberships are as follow: Senior 
Member, IEEE, Member IET, SIAM, ISA, OSA, SPIE, AIP and ISEBI. His current research interest is on advanced signal processing with applications in intelligent control system, biomedical and pharmaceutical systems, process control \& biotechnology, optical fiber sensors and microwave sensors.

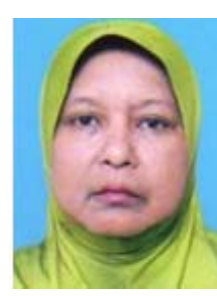

Noor Elaiza Abdul Khalid, has received BSc (Comp Sc) from USM, Malaysia in 1985, MSc (Comp Sc) in University of Wales in 1992 and $\mathrm{PhD}$ (Comp.Sc) from Universiti Teknologi MARA, Shah Alam in 2010. Currently hold position as senior lecturer in Faculty of Computer and Mathematical Sciences, Universiti Teknologi MARA, Shah Alam. Her current research interests are in Image Processing, Biomedical, Artificial Intelligence and Parallel Computing.

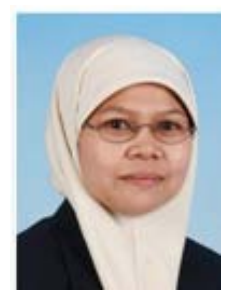

Haslina Taib is a Dental Lecturer from Unit of Peridontics School of Dental Sciences, Universiti Sains Malaysia. Pursued Master in Clinical Dentistry (Periodontology) from University of Malaya in 2006. Her research interest are periodontal bone loss and genetic. 